\title{
Finite Element and Higher order Difference Formulations for Modelling Heat Transport in Magnetised Plasmas
}

\author{
S. Günter, K. Lackner, C. Tichmann \\ Max-Planck Institut für Plasmaphysik, EURATOM-Association, 85748 Garching, Germany
}

\begin{abstract}
We present a finite element analogue to the second-order, finite difference scheme for the solution of the heat diffusion equation in strongly magnetised plasma given in Ref.[1]. Compared to standard finite element or finite difference formulations it strongly reduces the pollution of perpendicular heat fluxes by parallel ones even without resorting to field-aligned coordinates. We present both bi-linear and bi-quadratic versions of this scheme as well as a fourth-order extension of the original difference scheme of Ref.[1]. In the second part of the paper we address the formulation of the boundary conditions at walls with an oblique incidence of field lines and the treatment of the coordinate singularity at $r=0$ in cylindrical, or topologically equivalent coordinates with the reduced-pollution finite difference scheme. All tests shown indicate that both the finite-difference and the finite-element versions of the scheme should substantially alleviate the requirement for field-alignment of the coordinates over the realistic range of $\chi_{\|} / \chi_{\perp}$ in toroidal magnetic confinement devices.
\end{abstract}

\section{Introduction}

In reference [1] we presented a $2^{\text {nd }}$ order correct finite difference scheme for the solution of the heat conduction equation in strongly magnetised plasmas, which reduces significantly the pollution of perpendicular heat transport by numerical errors in the evaluation of the parallel one. Although a choice of the coordinate system aligned as well as possible with the magnetic flux surfaces or field lines remains indicated for a number of reasons outlined in the following, the scheme developed was shown to be tolerant to the violation of this requirement, even for completely unaligned coordinates, and values of the heat conductivity ratio up to $\chi_{\|} / \chi_{\perp}=10^{9}$ in plane and $10^{12}$ in periodic cylinder geometry. We compared the scheme with other schemes at hand of well-known 2-d test cases [1,2] in Cartesian coordinates, and showed 3-d applications to the problem of transport across magnetic islands and regions of ergodic field lines utilizing a combined finite difference - Fourier representation in cylinder coordinates.

A major criticism to the scheme was that is was not apparent how it could be utilized in the frame of a finite element representation, like used in most nonlinear MHD codes. We present in this paper such a formulation for bi-linear and bi-quadratic basis functions, and give also the extension of the finite difference version to $4^{\text {th }}$ order accuracy. In the second part we address two practical problems arising from the implementation of the physically proper boundary conditions at material walls and from the singularity arising at the axis in cylindrical or topologically equivalent coordinates. Unless indicated otherwise, we use the nomenclature and the labelling of grid points as in Fig.1 of Ref.[1] throughout this paper. 


\section{Generalisation of the scheme}

\subsection{Finite element formulation}

The difference scheme described in Ref. [1] and further developed below in this paper has been shown to be successful even in cases of extremely anisotropic heat transport problems. One main application for such a scheme are non-linear MHD simulations, where tolerance of a scheme to misalignment between coordinate and magnetic field lines would become a particular asset. As most of the existing non-linear MHD codes (see e.g. [2,3]) are based, however, on a finite element approach it is desirable to develop also a finite element scheme with similar properties as our finite difference formulation.

In a finite element framework the stationary heat conduction equation with constant heat diffusivities is reformulated as

$$
\int v_{i j} \nabla \cdot \vec{q} d V_{i j}=-\int \nabla v_{i j} \cdot \vec{q} d V_{i j}=\int n\left(\chi_{\|}-\chi_{\perp}\right)\left(\nabla v_{i j} \cdot \vec{b}\right)(\vec{b} \cdot \nabla T) d V_{i j}+\int n \chi_{\perp} \nabla v_{i j} \cdot \nabla T d V=\int v_{i j} Q d V_{i j}
$$

(for simplicity Dirichlet boundary conditions have been used) with the test functions $v_{i j}$. The temperature is approximated by a linear combination of local expansion functions

$$
T=\sum_{k l} v_{k l} T_{k l},
$$

and - in the usual case of conforming elements - derivatives are expressed through differentiation of the expansion functions. Calculations in Ref. [2] have shown, however, that this scheme requires high order elements to give satisfactory results for $\chi_{\|} / \chi_{\perp}$-ratios approaching realistic values.

To ensure a proper description of the heat flux parallel to the magnetic field lines already with lower-order finite elements, we investigated the use of a hybrid approach, defining a quantity $\tilde{q}_{\|}$through

$\int c_{i j}\left(\tilde{q}_{\|}-\vec{b} \cdot \nabla T\right) d V_{i j}=0$

taking the test functions $c_{i j}$ and the expansion functions for $\tilde{q}_{\|}$as one order lower than the local basis functions for the temperature $v_{i j}$. Instead of a separate representation of the two components of the heat flux $\chi_{\|} \nabla T$, projected point wise onto $\vec{b}$ this implies a finite element representation of $\tilde{q}_{/ /}$like a scalar quantity. As usual in hybrid finite element schemes, the corresponding expansion and test functions for $\tilde{q}_{/ /}$are discontinuous at the element borders. The difference between the two representations corresponds to a truncation error contribution of the same order as the one implied by the choice of elements for $T$, and should hence not dominate its error scaling. On the other hand this special treatment of the parallel heat flux appears essential for allowing the parallel temperature gradient to vanish exactly for $\chi_{\|} / \chi_{\perp} \rightarrow \infty$. The choice bears analogy to our finite difference treatment, where it was found essential to define both components of the heat flux vector $\vec{q}_{/ /}$in the same grid points and 
express them through the same temperature grid values to greatly reduce numerical errors associated with anisotropy. The heat transport equation is then formulated like:

$$
\int v_{i j} \nabla \cdot \vec{q} d V_{i j}=-\int \nabla v_{i j} \cdot \vec{q} d V_{i j}=\int n\left(\chi_{\|}-\chi_{\perp}\right)\left(\nabla v_{i j} \vec{b}\right) \tilde{q}_{\|} d V_{i j}+\int n \chi_{\perp} \nabla v_{i j} \cdot \nabla T d V=\int v_{i j} Q d V_{i j}
$$

We have implemented this scheme using both bilinear and bi-quadratic test functions $v_{i j}$ for $T$ over quadrilateral elements, using Gaussian integration of appropriate order to evaluate the integrals. The expansion functions $c_{i j}$ of $\tilde{q}_{\|}$in the two cases become constants or bilinear functions, respectively. We compared our hybrid approach with the standard one with conforming finite elements, using an own code for the bilinear case, and results of Ref. [2] covering up to bi-quintic finite elements. As test case we use the NIMROD test problem, already used by us in Ref. [1], which allows an analytic solution: taking a 2-d magnetic field configuration derived from a flux function as $\vec{B}=\vec{e}_{z} \times \nabla \psi$, and choosing a heat source satisfying $Q(x, y)=-\chi_{\perp} \nabla^{2} \psi(x, y)$, ensures, together with appropriate boundary conditions, that the true solution will be a constant on field lines, irrespective of the value of $\chi_{\|} / \chi_{\perp}$ [2]. The functional form chosen for $\psi(x, y), T(x, y)$ in our case is $\sin (\pi x) \cdot \cos (\pi y)$, over the interval $-0.5 \leq x, y \leq 0.5$, together with Dirichlet boundary conditions. The relative errors quoted refer to the temperature value at the centre: $\left|T(0,0)^{-1}-1\right|$, corresponding also to the magnetic axis. Fig. 1 shows the results for the NIMROD test problem with the lowest order implementation of the above scheme. As for the finite difference scheme, the numerical errors are found not depend on $\chi_{\|} / \chi_{\perp}$ and are similar to those of our second order accurate finite difference scheme.

Results shown in Figs 1 a $-\mathrm{c}$ correspond to $\chi_{\|} / \chi_{\perp}$-ratios of $10^{3}, 10^{6}$ and $10^{9}$, respectively. Already for the lowest value of this ratio, both the bi-linear and the bi-quadratic versions of the hybrid scheme have errors which are one to two orders of magnitude smaller than their conforming counterparts. For $\chi_{\|} / \chi_{\perp}=10^{6}$ conforming bilinear elements become totally unacceptable. Bi-linear hybrid elements perform already better than bi-quadratic conforming ones and bi-quadratic hybrid better than conforming bi-cubic ones. This trend is further increased going to higher values of $\chi_{\|} / \chi_{\perp}$, as the error for the hybrid element calculations practically does not change between $10^{6}$ and $10^{9}$, whereas it increases strongly for the conforming element cases.

\subsection{A fourth order finite difference formulation}

The finite difference scheme described in Ref. [1] is accurate to second order only. It can be readily extended to fourth order; however, as we show here for the case of Cartesian coordinates in two dimensions. It is based on an expansion of the temperature up to third order in each direction:

$$
\begin{aligned}
& T(\Delta x, \Delta y)=T(0,0)+a_{10} \Delta x+a_{01} \Delta y+a_{11} \Delta x \Delta y+a_{20}(\Delta x)^{2}+a_{02}(\Delta y)^{2}+a_{12} \Delta x(\Delta y)^{2}+a_{21}(\Delta x)^{2} \Delta y \\
& \quad+a_{22}(\Delta x)^{2}(\Delta y)^{2}+a_{13} \Delta x(\Delta y)^{3}+a_{31}(\Delta x)^{3} \Delta y+a_{23}(\Delta x)^{2}(\Delta y)^{3}+a_{32}(\Delta x)^{3}(\Delta y)^{2}+a_{33}(\Delta x)^{3}(\Delta y)^{3}
\end{aligned}
$$


where we define - as in Ref. [1]- the temperatures at the grid points and the heat fluxes at intermediate grid points. Again we use the freedom of adding terms leading to higher order ( $5^{\text {th }}$ and above) corrections to ensure that both components of the parallel heat flux are defined in the same grid points. This leads to the expression

$$
\begin{aligned}
\vec{q}_{i+1 / 2, j+1 / 2}^{\|}=-n \chi_{\|} \vec{b} \cdot\left(\vec{b} \cdot \nabla_{x} T+\vec{b} \cdot \nabla_{y} T\right)_{i+1 / 2, j+1 / 2} \\
\left.=-n \chi_{\|}\left\{b^{x}, b^{y}\right\}\left(\begin{array}{l}
\left.\frac{b_{i+1 / 2, j+1 / 2}^{x}}{384 \Delta x}\left(\begin{array}{l}
243\left(T_{i+1, j+1}+T_{i+1, j}-T_{i, j+1}-T_{i, j}\right)+27\left(T_{i, j+2}+T_{i, j-1}-T_{i+1, j+2}-T_{i+1, j-1}\right) \\
+9\left(T_{i-1, j+1}+T_{i-1, j}-T_{i+2, j+1}-T_{i+2, j}\right)+\left(T_{i+2, j+2}+T_{i+2, j-1}-T_{i-1, j+2}-T_{i-1, j-1}\right.
\end{array}\right)\right)+ \\
+\frac{b_{i+1 / 2, j+1 / 2}^{y}}{384 \Delta y}\left(\begin{array}{l}
243\left(T_{i+1, j+1}-T_{i+1, j}+T_{i, j+1}-T_{i, j}\right)-9\left(T_{i, j+2}-T_{i, j-1}+T_{i+1, j+2}-T_{i+1, j-1}\right) \\
-27\left(T_{i-1, j+1}-T_{i-1, j}+T_{i+2, j+1}-T_{i+2, j}\right)+\left(T_{i+2, j+2}-T_{i+2, j-1}+T_{i-1, j+2}-T_{i-1, j-1}\right.
\end{array}\right)
\end{array}\right)\right)
\end{aligned}
$$

for the parallel heat flux and to

$$
\begin{aligned}
\left.\nabla \cdot \vec{q}^{\|}\right|_{i, j}=\frac{1}{384}\left\{\begin{array}{l}
\left\{\vec{e}_{x}, 0\right\} \\
\Delta x
\end{array}\left(\begin{array}{l}
243\left(\vec{q}_{i+1 / 2, j+1 / 2}^{\|}+\vec{q}_{i+1 / 2, j-1 / 2}^{\|}-\vec{q}_{i-1 / 2, j+1 / 2}^{\|}-\vec{q}_{i-1 / 2, j-1 / 2}^{\|}\right) \\
+27\left(\vec{q}_{i-1 / 2, j+3 / 2}^{\|}+\vec{q}_{i-1 / 2, j-3 / 2}^{\|}-\vec{q}_{i+1 / 2, j+3 / 2}^{\|}-\vec{q}_{i+1 / 2, j-3 / 2}^{\|}\right) \\
+9\left(\vec{q}_{i-3 / 2, j+1 / 2}^{\|}+\vec{q}_{i-3 / 2, j-1 / 2}^{\|}-\vec{q}_{i+3 / 2, j+1 / 2}^{\|}-\vec{q}_{i+3 / 2, j-1 / 2}^{\|}\right) \\
+\left(\vec{q}_{i+3 / 2, j+3 / 2}^{\|}+\vec{q}_{i+3 / 2, j-3 / 2}^{\|}-\vec{q}_{i-3 / 2, j+3 / 2}^{\|}-\vec{q}_{i-3 / 2, j-3 / 2}^{\|}\right)
\end{array}\right)\right. \\
\left.+\frac{\left\{0, \vec{e}_{y}\right\}}{\Delta y}\left(\begin{array}{l}
243\left(\vec{q}_{i+1 / 2, j+1 / 2}^{\|}-\vec{q}_{i+1 / 2, j-1 / 2}^{\|}+\vec{q}_{i-1 / 2, j+1 / 2}^{\|}-\vec{q}_{i-1 / 2, j-1 / 2}^{\|}\right) \\
-9\left(\vec{q}_{i-1 / 2, j+3 / 2}^{\|}-\vec{q}_{i-1 / 2, j-3 / 2}^{\|}+\vec{q}_{i+1 / 2, j+3 / 2}^{\|}-\vec{q}_{i+1 / 2, j-3 / 2}^{\|}\right) \\
-27\left(\vec{q}_{i-3 / 2, j+1 / 2}^{\|}-\vec{q}_{i-3 / 2, j-1 / 2}^{\|}+\vec{q}_{i+3 / 2, j+1 / 2}^{\|}-\vec{q}_{i+3 / 2, j-1 / 2}^{\|}\right) \\
+\left(\vec{q}_{i+3 / 2, j+3 / 2}^{\|}-\vec{q}_{i+3 / 2, j-3 / 2}^{\|}+\vec{q}_{i-3 / 2, j+3 / 2}^{\|}-\vec{q}_{i-3 / 2, j-3 / 2}^{\|}\right)
\end{array}\right)\right\}
\end{aligned}
$$

for its divergence.

We have tested this fourth-order scheme again for the above test case proposed by the NIMROD team. To avoid the problem of the specification of additional (unphysical) boundary conditions for the $4^{\text {th }}$ order scheme, we reduce the formulation to the second-order one described in Ref. [1] over the outermost two grid cells. Fig. 2 gives the results for values of $\chi_{\|} / \chi_{\perp}=10^{6}$ and $10^{9}$ for both the second and the fourth order scheme, showing the expected improvement by the latter (except for the lowest resolution case, where the fourthorder schemes error is dominated by the implementation of the boundary conditions). The discretisation error for both schemes is again independent of the ratio $\chi_{\|} / \chi_{\perp}$. For the higher numbers of grid points and high values of $\chi_{\|} / \chi_{\perp}$, the fourth order scheme becomes, however, very susceptible to round-off errors, requiring improved (16 instead of 8-byte) precision to realize its potential accuracy. 


\section{Treatment of general boundary conditions and coordinate singularities}

The test cases presented in Ref. [1] used Dirichlet boundary conditions, and were formulated in either Cartesian coordinates or in cylindrical coordinates using, in the latter case a Fourier description in both poloidal $(\theta-)$ and axial $(z-)$ direction. Complications arise in the more general case, when prescriptions involving the conductive heat flux have to be applied at the boundary, or if cylindrical (or topologically equivalent) coordinates are used in 2 or 3-d finite difference formulations including the point $r=0$. In this section we demonstrate that the developed finite difference scheme is able to deal with such realistic boundary conditions.

\subsection{Realistic boundary conditions for magnetised plasmas}

In the general case flux surfaces or field lines will cross the computational boundary. The boundary conditions to be applied in that case will be of a mixed (Robin-) type and will typically be dominated by the physics of the parallel heat flux. In particular, if the walls are assumed to correspond to material surfaces the boundary conditions will be given by the wellknown Bohm-type condition [4,5]:

$-\vec{b} \cdot \vec{e}_{w} n_{e} \chi_{\|} \vec{b} \cdot \nabla T=\delta_{\|} n_{e} c_{s} k_{B} T \vec{b} \cdot \vec{e}_{w}$,

where $\vec{e}_{w}$ is the unit vector perpendicular to the wall, pointing outward, $\vec{b}$ the unit vector along the field lines, $\delta_{\|}$the so-called sheath transmission factor and $c_{s}$ the ion sound speed. For field lines nearly tangent to the walls the much smaller perpendicular heat transport has also to be included to remove the singularity otherwise arising for $\vec{b} \cdot \vec{e}_{w}=0$. We therefore write, in the general case the boundary condition in the form

$-\vec{b} \cdot \vec{e}_{w}\left(\chi_{\|} / \chi_{\perp}-1\right) \vec{b} \cdot \nabla T-\vec{e}_{\perp} \cdot \nabla T=\delta_{e f f} T\left(\vec{b} \cdot \vec{e}_{w}+\varepsilon_{\perp}\right)$

where $\delta_{\text {eff }}=\delta_{/ /} c_{s} k_{B} / \chi_{\perp}$, and $\varepsilon_{\perp}$ is a usually small number characterising the heat flux into the wall perpendicular to field lines.

The above boundary conditions are of Robin type, which relate derivative and function values at the same grid point. As in our scheme the gradients are defined in the intermediate grid points only, we express them at the boundary points $(i, j)$ by:

$$
\begin{aligned}
\left.\nabla T\right|_{i, j} \cdot \vec{e}_{x} & =\frac{1}{2}\left(\left.\nabla T\right|_{i-1 / 2, j-1 / 2} \cdot\left\{\vec{e}_{x}, \vec{e}_{y}\right\}+\left.\nabla T\right|_{i-1 / 2, j+1 / 2} \cdot\left\{\vec{e}_{x},-\vec{e}_{y}\right\}\right) \\
& =\frac{\left(T_{i, j}+T_{i, j-1}\right)-\left(T_{i-1, j}+T_{i-1, j-1}\right)}{4 \Delta x} \cdot\left\{\vec{e}_{x}, \vec{e}_{y}\right\}+\frac{\left(T_{i, j}+T_{i, j+1}\right)-\left(T_{i-1, j}+T_{i-1, j+1}\right)}{4 \Delta x} \cdot\left\{\vec{e}_{x},-\vec{e}_{y}\right\}
\end{aligned}
$$

A useful test example is given by the magnetic field configuration used in Fig. 3, which shows a poloidal cut through the separatrix- limited plasma of a divertor tokamak, whose divertor plates coincide with sections of the rectangular computational boundary. For generality, a geometry with two X-points, lying on non-coincident magnetic flux surfaces, is chosen. A heat source, spatially constant over the whole computational domain was assumed 
for this test case. This formulation, together with the above-mentioned boundary conditions, and realistically high values of $\chi_{\| /} / \chi_{\perp}$ results in the formation of a thin, nearly flux-surface aligned boundary layer (the "scrape-off layer" [6] -SOL) along the inner separatrix. In codes addressing specifically transport in the edge region ([7-9]), this zone would typically be treated using coordinates aligned with the flux-surfaces. Using simple cylindrical coordinates $r, z, \varphi$, with $\varphi$ the (ignorable) toroidal coordinate is therefore a realistically severe test case for our present scheme.

Realistic values of heat conductivity in the edge region of tokamak plasmas correspond to ratios of $\chi_{\|} / \chi_{\perp}$ in the range of $10^{8}$. As we make use of the toroidal symmetry which reduces the problem to a 2d geometry, an effective heat conductivity ratio $\chi_{\|} / \chi_{\perp} \cdot\left(B_{r}^{2}+B_{z}^{2}\right) /\left(B_{r}^{2}+B_{z}^{2}+B_{\varphi}^{2}\right)$ enters actually into the calculations as a measure of the anisotropy. The poloidal to toroidal field ratio $\sqrt{\left(B_{r}^{2}+B_{z}^{2}\right) /\left(B_{r}^{2}+B_{z}^{2}+B_{\varphi}^{2}\right)}$ in a given tokamak varies over space (in particular vanishing at the axis and the stagnation point on the separatrix) and depends also on operating parameters. For the case shown here, its value at a reference point taken at the intersection of the outside separatrix with the mid-plane was 0.11 .

The temperature distribution calculated like this is shown in Fig. 3 in the form of isocontours. They show the expected formation of two scrape-off layers around the two separatrix flux surfaces. The vanishing of the effective heat conductivity ratio $\chi_{\|} / \chi_{\perp}\left(B_{r}^{2}+B_{z}^{2}\right) /\left(B_{r}^{2}+B_{z}^{2}+B_{\varphi}^{2}\right)$ in the proximity of the stagnation points causes there visibly significant temperature gradients along the flux surfaces open towards the wall. (At the lower stagnation point, even a local maximum of $T$ arises, which is, however, a - numerically correct - consequence of our unphysical assumption of a spatially constant heat source also outside the separatrix).

A cross-sectional cut of the temperature profile across the $z=0$ plane shows (Fig. 4) that the scrape-off layer region is the numerically most demanding region of the calculations. In fact, a minimum number of grid points is required to resolve it, as otherwise oscillations arise. This is clearly a strong argument for using a coordinate system allowing grid meshing in this region (or using a non-structured grid), which would of course be the case if flux surface coordinates were to be used.

Without an adapted grid, use of a higher order scheme for this problem is not advantageous. The existence of the scrape-off layer introduces - albeit over a limited space region - a characteristic dimension perpendicular to flux surfaces which has to be resolved by a sufficient number of grid points. This annihilates most of the potential benefit of a higher order scheme, as one cannot use it to increase significantly the grid interval.

\subsection{Use of cylindrical coordinates including the $r=0$ coordinate singularity}

In practice, even for the above 2-d example one would therefore use a coordinate system which is aligned at least approximately with the magnetic flux surfaces. Apart from advantages deriving from a better separation of parallel and perpendicular heat fluxes, this would allow also to apply meshing around critical flux surfaces, like - for the above example - around the separatrix region. Such coordinate systems share with cylinder coordinates (taken 
now in $r, \theta, z$, with $\mathrm{z}$ substituting the toroidal direction) the property that a coordinate singularity appears at $r=0$. To clarify the issues arising form this coordinate singularity we can use polar coordinates $(r, \theta)$, with the point $r=0$ inside the computational region. The corresponding parallel heat fluxes, defined again at the intermediate grid points, are given by:

$$
\begin{aligned}
\vec{q}_{i+1 / 2, j+1 / 2}^{\|} & =-n \chi_{\|} \vec{b} \cdot\left(\vec{b} \cdot \nabla_{r} T+\vec{b} \cdot \nabla_{\theta} T\right)_{i+1 / 2, j+1 / 2} \\
& =-n \chi_{\|}\left\{b^{r}, b^{\theta}\right\}\left(\begin{array}{l}
b_{i+1 / 2, j+1 / 2}^{r} \frac{\left(T_{i+1, j+1}+T_{i+1, j}-T_{i, j+1}-T_{i, j}\right)}{2 \Delta r}+ \\
+b_{i+1 / 2, j+1 / 2}^{\theta} \frac{\left(T_{i+1, j+1}+T_{i, j+1}-T_{i+1, j}-T_{i, j}\right)}{2 \Delta \theta r_{i+1 / 2, j+1 / 2}}
\end{array}\right)
\end{aligned}
$$

The divergence of the heat flux (except in the central point) is defined by:

$$
\begin{aligned}
\left.\nabla \cdot \vec{q}\right|_{i, j}=\frac{1}{2 r_{i, j}}\left\{\frac{\left\{\vec{e}_{r}, 0\right\}}{\Delta r}\left(\begin{array}{l}
r_{i+1 / 2, j+1 / 2} \vec{q}_{i+1 / 2, j+1 / 2}^{\|}+r_{i+1 / 2, j-1 / 2} \vec{q}_{i+1 / 2, j-1 / 2}^{\|} \\
-r_{i-1 / 2, j+1 / 2} \vec{q}_{i-1 / 2, j+1 / 2}^{\|}-r_{i-1 / 2, j-1 / 2} \vec{q}_{i-1 / 2, j-1 / 2}^{\|}
\end{array}\right)\right. \\
\left.+\frac{\left\{0, \vec{e}_{\theta}\right\}}{\Delta \theta}\left(\vec{q}_{i+1 / 2, j+1 / 2}^{\|}+\vec{q}_{i-1 / 2, j+1 / 2}^{\|}-\vec{q}_{i+1 / 2, j-1 / 2}^{\|}-\vec{q}_{i-1 / 2, j-1 / 2}^{\|}\right)\right\}
\end{aligned}
$$

These expressions show, of course, a singular behaviour at the central points $r_{l, \mathrm{j}}=r=0$. To eliminate this singularity, we derive the equation for $r=0$ from integration of the heat flux equation over the volume inside $r_{1 / 2}$

$$
\int_{0}^{2 \pi} d \theta \int_{0}^{r_{1} / 2} r d r \nabla \cdot \vec{q}
$$

That way we find the following result for the equation for $r=0$ :

$$
\left(\chi_{\|}-1\right) \frac{2}{r_{1 / 2}} \frac{1}{N_{\theta}} \sum_{j=1}^{N_{\theta}}\left\{\vec{e}_{r}, 0\right\} \vec{q}_{1 / 2, j-1 / 2}^{\|}+\chi_{\perp} \frac{2}{r_{1 / 2}} \frac{1}{N_{\theta}} \sum_{j=1}^{N_{\theta}} q_{1 / 2, j-1 / 2}^{\perp}=Q_{0}
$$

A simple, but severe test for this procedure can be constructed considering both circular coordinate and flux surfaces, but shifting the origin of the two with respect to the each other. For the present example we use a magnetic field described by the magnetic flux function: $\Psi(r, \theta)=\left((r \cos \theta-0.1)^{2}+r^{2} \sin ^{2} \theta\right)^{3 / 2}$ with magnetic field components defined by $b_{r}=\frac{1}{r} \frac{\partial \Psi}{\partial \theta}, b_{\theta}=-\frac{\partial \Psi}{\partial r}$, and a heat source given by $Q=9 \cdot(\Psi(r, \theta))^{1 / 3}$, looking for a solution in the region $(0 \leq r \leq 1,0 \leq \theta \leq 2 \pi)$. The boundary conditions at $r=1$ are taken as Dirichlet conditions using the known analytical solution $T(r, \theta)=1-\Psi(r, \theta)$. The consistency of this procedure is illustrated by the temperature profile taken at $\sin \theta=0$ given in Fig. 5, which shows no visible influence of the coordinate singularity at $r=0$ on the solution. The scaling of the error with grid size (Figure 6) is affected by several effects, in particular also by the intersection of the boundary by the field lines and the imposition of the analytical solution on 
it, which has stronger effect for high $\chi_{\|} / \chi_{\perp}$. Probably for this reason, the numerical error for a modest number of grid points is actually smaller in the case of higher $\chi_{\|} / \chi_{\perp}$, but approaches the results for lower values of $\chi_{\|} / \chi_{\perp}$ if a sufficient number of grid points is taken.

One prime reason for the choice of (approximately) aligned coordinates is the easy possibility of effective meshing. To illustrate this in the context also of the finite difference formulation in $r, \theta$ (it had been shown in Ref.[1] already for the case of Fourier decomposition in the poloidal angle $\theta$ ) in a simple model we consider a cylindrical 2-d plasma with flux surfaces given by $\Psi(r, \theta)=\left(r^{2}-r_{s}^{2}\right)^{2}+0.02 r^{2}\left(1-r^{2}\right) \cos \theta$ producing a magnetic island at $r_{s}=0.6$. (Comparing these cases to the physically more realistic model of a single helicity resonant perturbation of an infinite aspect ratio, circular cross-section tokamak, which can also be treated as rigorously 2 -d, one has to multiply the values of $\chi_{\|} / \chi_{\perp}$ used here by a factor of $\left(\frac{q_{s}}{\varepsilon} \cdot \frac{q}{q_{s}-q}\right)^{2}$, where $q$ and $q_{s}$ are the local and resonant values of the so-called safety factor $q=\varepsilon \frac{B_{\varphi}}{B_{\theta}}$, and $\varepsilon$ the local aspect ratio. This factor typically is of $\left.O\left(10^{2}\right)\right)$. The heat source used is $Q=15000 r^{3}-4500 r^{2}+60$ for $r \leq 0.2$ and $Q=0$ beyond. In a radial cut through the O-point of the island (Fig. 7), the high parallel heat conductivity results in the formation of a plateau in the temperature profile, with very large radial second derivatives at its boundaries. Like in the problem of Figs. 3,4 these regions have to be well resolved, which can be accomplished with less effort if meshing in the corresponding regions of $r$ is used. The plot of the global temperature profile shows hardly a dependence on the grid resolution, but a closer inspection of the near- separatrix region of the magnetic island, or a plot of the local error at the most sensitive point (Fig. 8) shows an improvement with grid number or meshing. As the thickness of the boundary layer forming around the island separatrix decreases with increasing $\chi_{\|} / \chi_{\perp}$, the resolution requirements, respectively the error increase with this parameter.

\section{Conclusions}

We have shown how to transfer the basic concept of the scheme presented in Ref. [1] for the treatment of the heat conduction problem in strongly magnetized plasmas also to a finite element formulation. The favourable properties regarding the suppression of pollution of the perpendicular by the parallel heat transport can be conserved, provided a proper hybrid formulation is used. Both the finite difference scheme of Ref. [1] and the finite element formulation of the present paper can be readily extended also to higher orders, albeit requiring in this case - for large values of $\chi_{\|} / \chi_{\perp}$ and a large number of grid points - the use of higher precision in the matrix solver.

The favourable pollution properties, however, do not eliminate the need to resolve spatially well the regions with large second derivatives in $T$ perpendicular to the flux surfaces, arising, in particular in boundary layers around the separatrix of divertor tokamaks, or around magnetic islands at resonant flux surfaces. This gives a strong motivation to use coordinates, at least approximately adapted to the flux surfaces, to allow localized fine-meshing of the 
grid. If, in addition, one coordinate line $x^{1}$ is chosen so as to follow approximately the field lines, the effective ratio of $\chi_{\|} / \chi_{\perp}$ is reduced by a factor $\left(\vec{B} \times \nabla x^{1}\right)^{2} /(\vec{B} \cdot \vec{B})$. The benefit of our present scheme in the latter case is the greater tolerance to misalignment between $\vec{B}$ and $\nabla x^{1}$, making it well suited to nonlinear MHD codes - where it would be excessively cumbersome to adjust the coordinate system continuously to the magnetic field configuration - and to problems including regions of ergodised field lines. This feature has in fact been already exploited in Ref. [10], for the study of heat transport in the magnetic field of an infinite aspect ratio tokamak with circular plasma cross-section perturbed by several helical perturbations, using - in contrast to Ref. [1] - a 2-d grid and a Fourier representation of the perturbations in the $3^{\text {rd }}$ direction.

\section{Appendix: Boundary conditions for heat conduction equation at a wall}

\section{Figure Captions:}
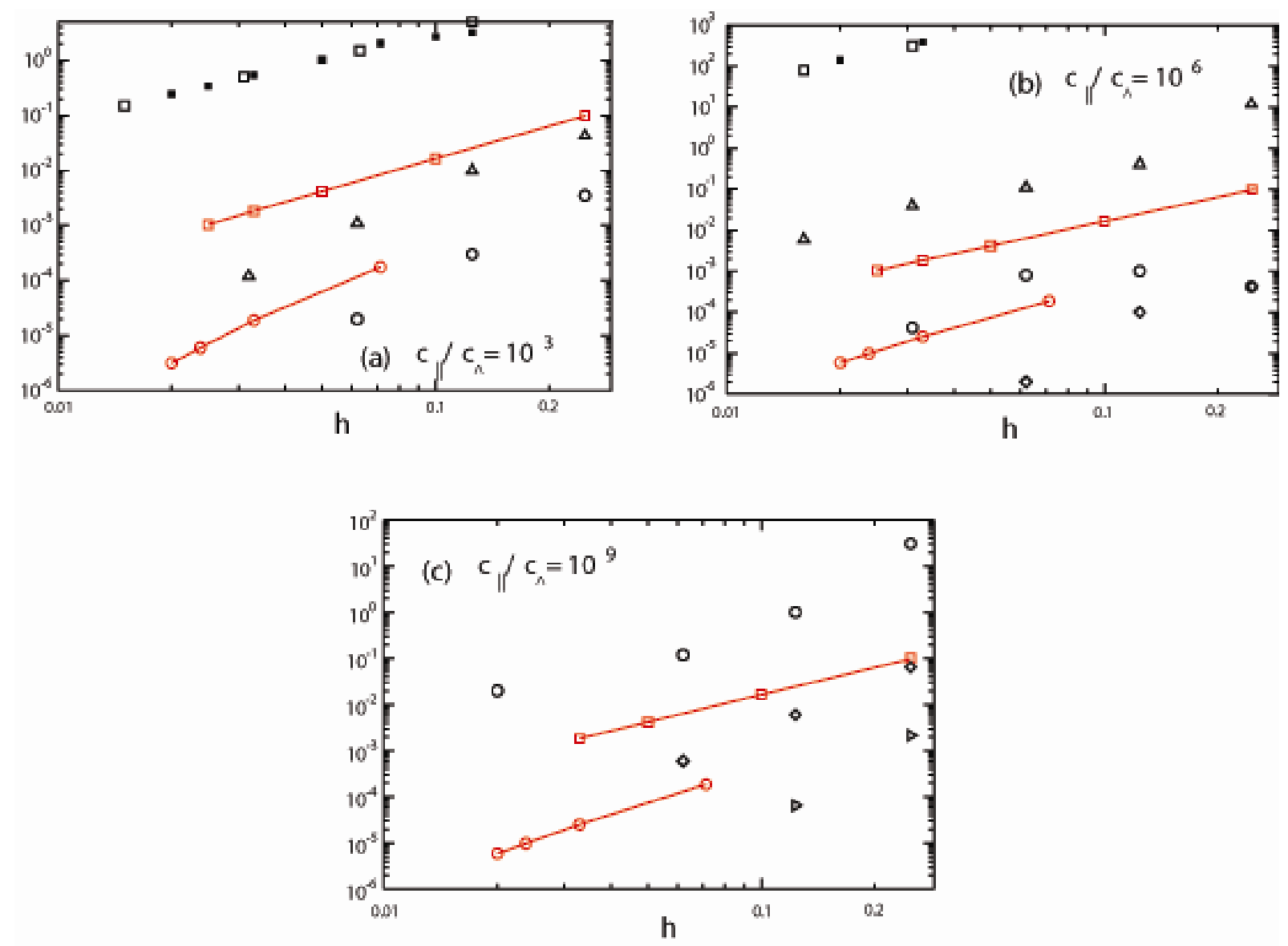

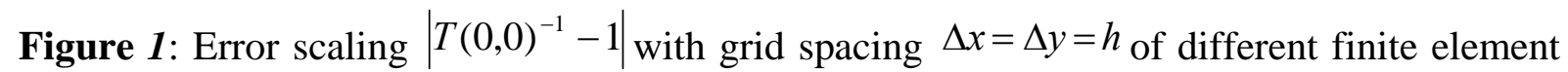
schemes for the NIMROD test case, for values of $\chi_{\|} / \chi_{\perp}=10^{3}$ (Fig.1a), $10^{6}$ (1b) and $10^{9}$ (1c). Shown are bi-linear conforming elements, using both results of our own code (full black 
squares) and those of the authors of Ref. [2] (open black squares), and results of Ref.[2] with bi-quadratic (open black triangles), bi-cubic (open black circles) and bi-quintic (open black diamonds) conforming elements, compared to the hybrid element schemes with bi-linear (red open squares) and bi-quadratic (red open circles) basis functions.

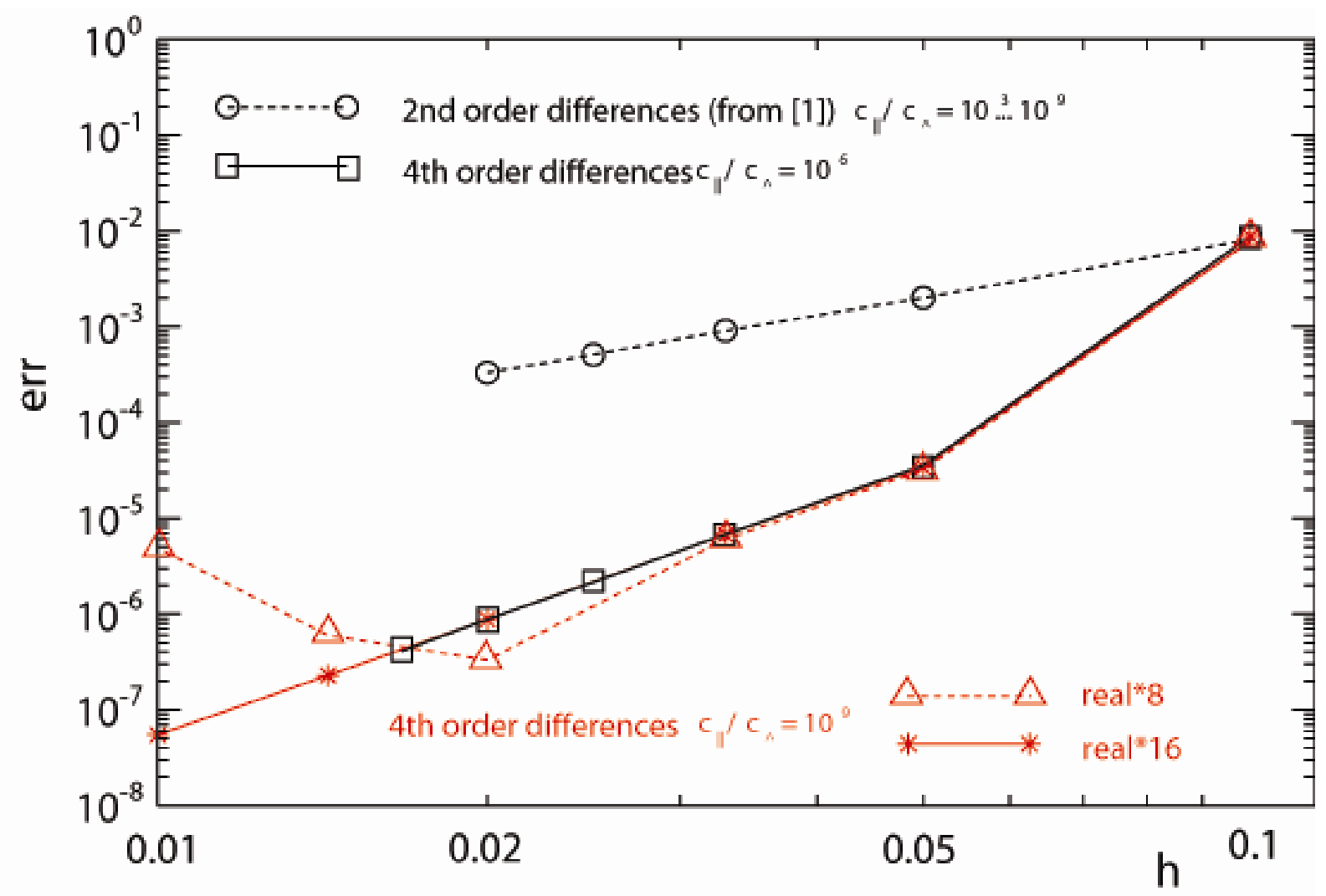

Figure 2: Error scaling $\left|T(0,0)^{-1}-1\right|$ with grid spacing of the $2^{\text {rd }}$ and $4^{\text {th }}$ - order difference scheme for the NIMROD test case for different values of $\chi_{\|} / \chi_{\perp}$. For the second order scheme, the error is virtually independent of this ratio in the range $10^{3}-10^{9}$. For the fourth order scheme, round-off errors start becoming important for small grid intervals and high values of $\chi_{\|} / \chi_{\perp}$, if standard double precision is used. With improved precision the independence of the error from $\chi_{\|} / \chi_{\perp}$ in the tested range is recovered. 


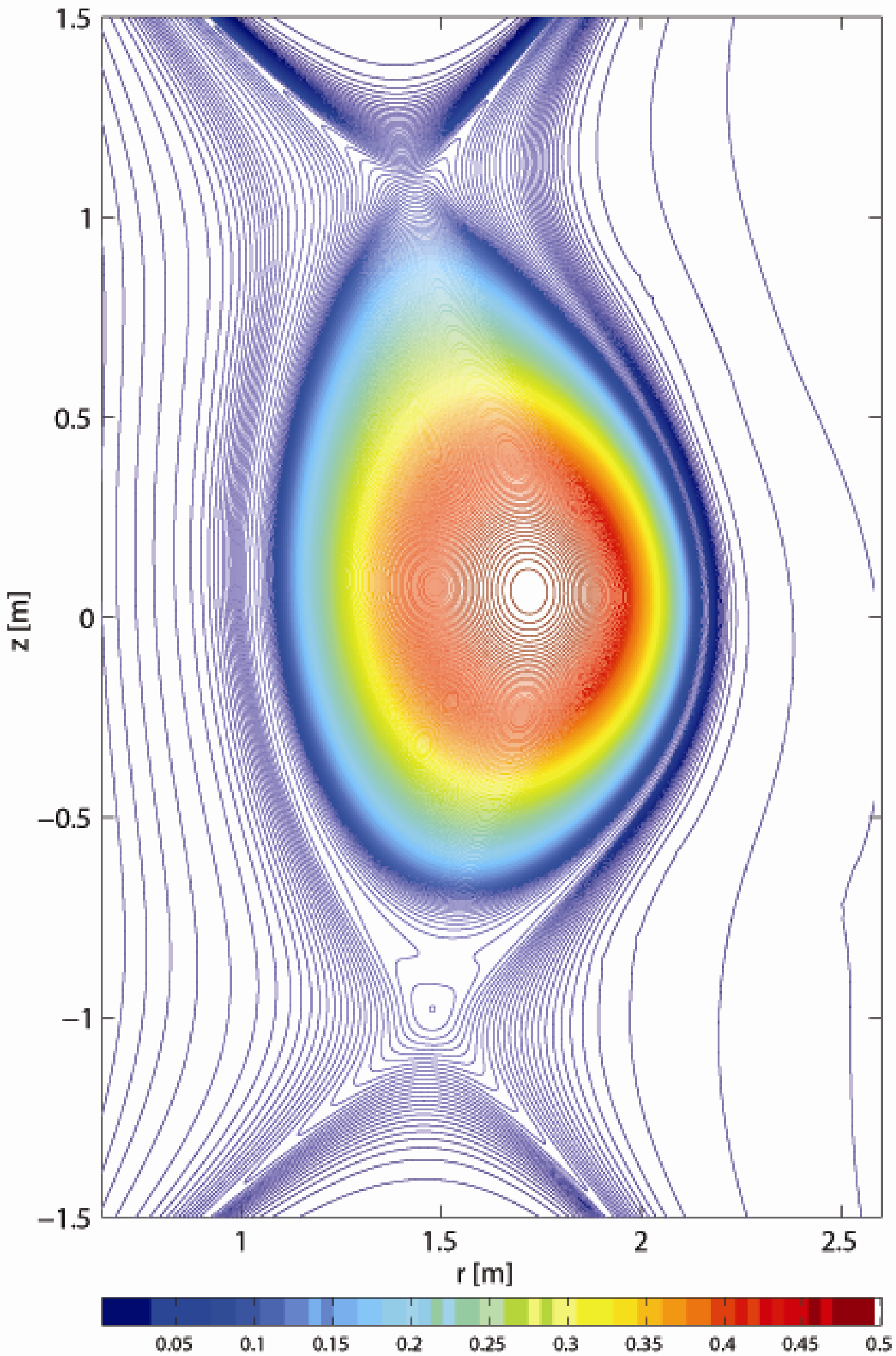

Figure 3: Temperature iso-contours (arbitrary units) in the poloidal cross-section of a divertor tokamak with assumed spatially constant heat source, computed with a second-order correct finite difference scheme and equidistant grid in $r, z$ with $1024 \times 2048$ grid points, $\chi_{\|} / \chi_{\perp}=10^{8}, \delta_{\text {eff }}=10^{4}, \varepsilon_{\perp}=10^{-3}$. The - spatially varying - ratio of the poloidal to toroidal 
magnetic field, determining the effective value of $\chi_{\|} / \chi_{\perp}$ in the two dimensions is $\sqrt{\left(B_{r}^{2}+B_{z}^{2}\right) /\left(B_{r}^{2}+B_{z}^{2}+B_{\varphi}^{2}\right)}=0.11$ at the outer outboard separatrix at the midplane $(r=2.22, z=0)$. The locations of the two inner and outer separatrices at the outboard midplane are indicated by the vertical lines.

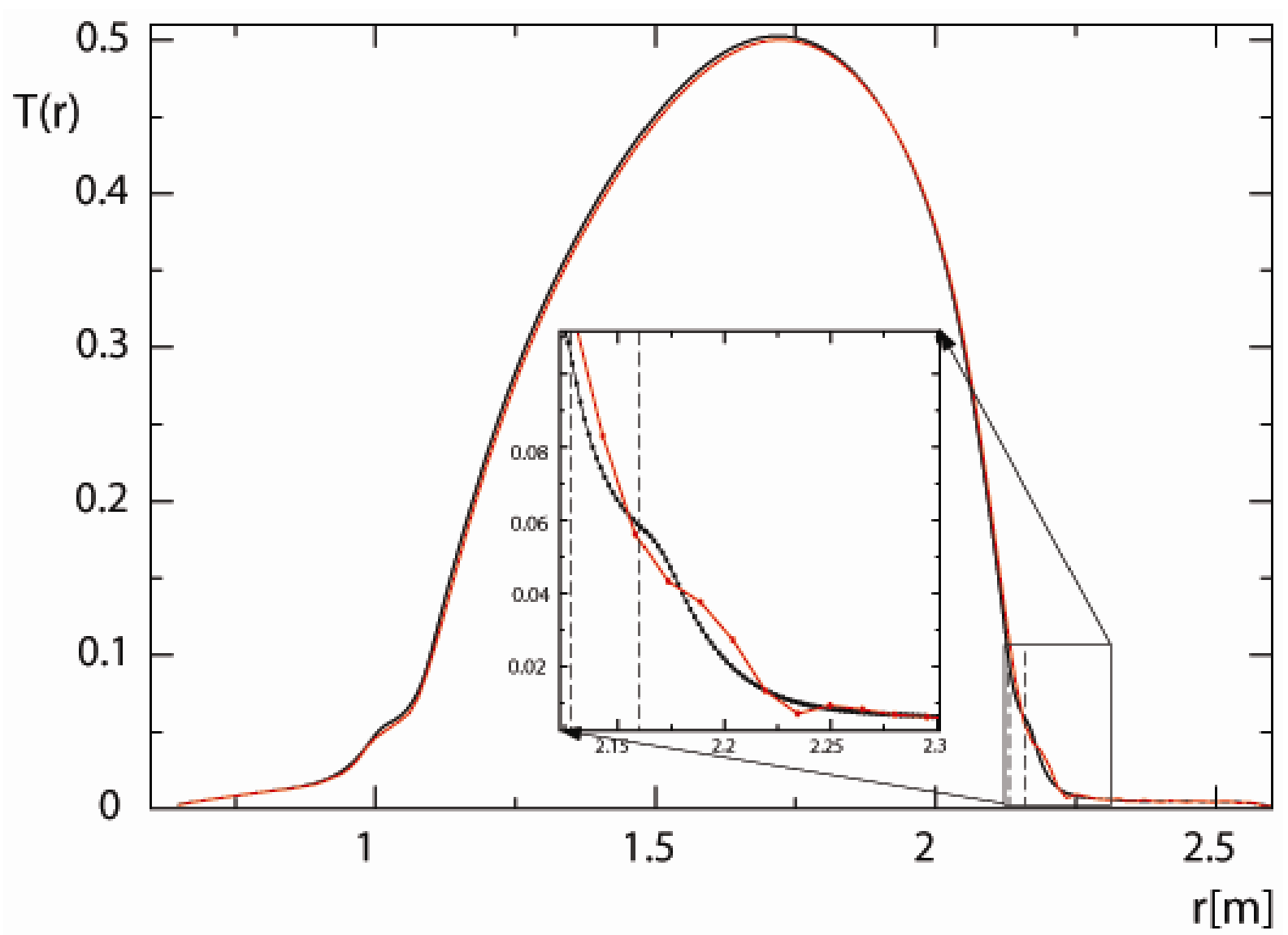

Figure 4: Radial temperature profile at $z=0$ for the case shown in Fig. 3, for computational grids with $128 \times 256$ and $1024 \times 2048$, respectively. Zoom shows the proximity of the intersection with the two separatrices, where the largest $2^{\text {nd }}$ derivatives of the temperature occur. A sufficient number of grid points over this region is needed to suppress local oscillations of the solution. 


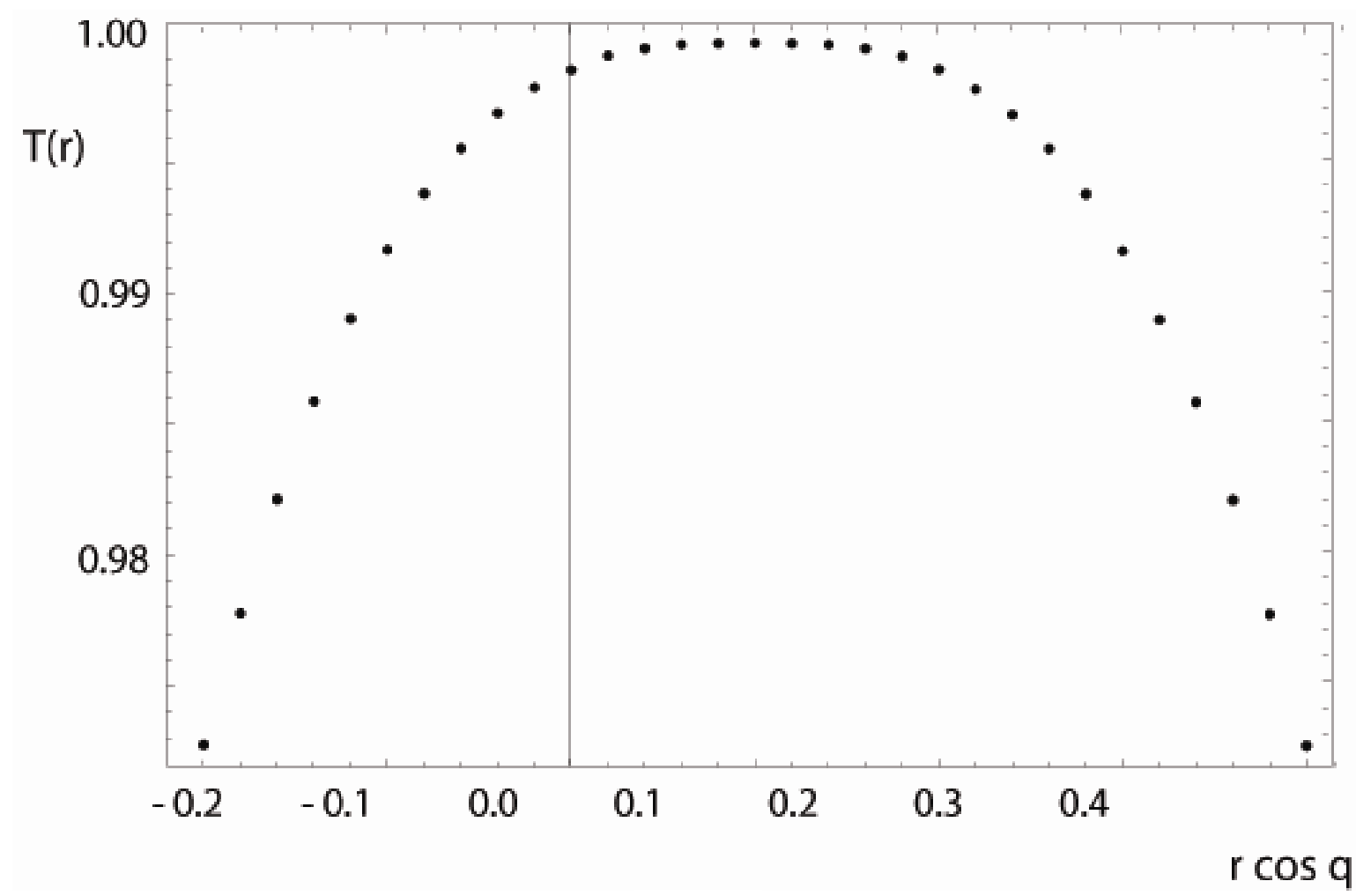

Figure 5: Temperature profile across the $\sin \theta=0$ line for a test example computed in cylindrical $r, \theta$-coordinates, with circular flux surfaces displaced with respect to the coordinate origin. Case shown refers to $50 \times 150$ grid points in $r, \theta$, respectively, and $\chi_{\|} / \chi_{\perp}=10^{9}$.

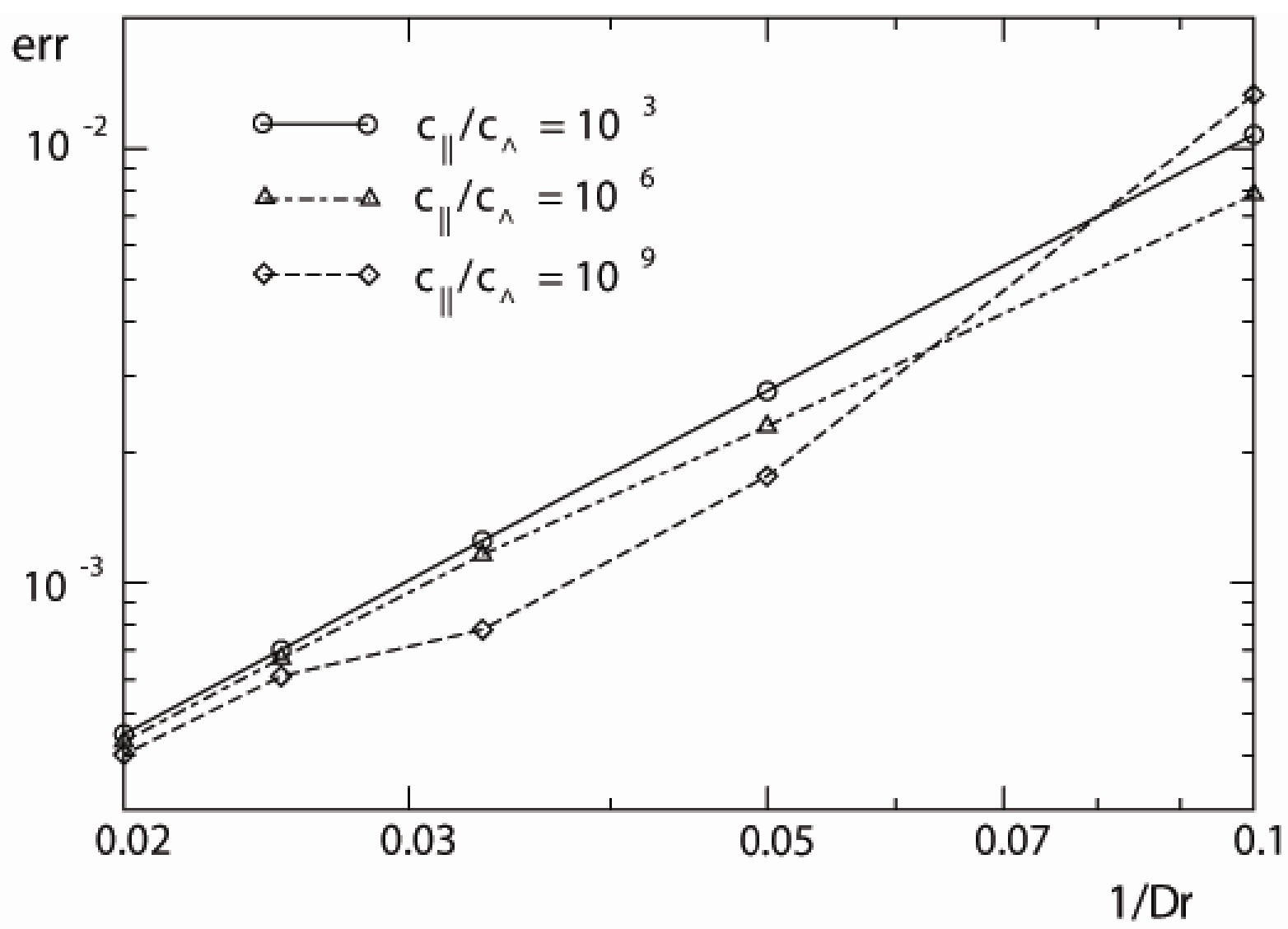


Figure 6: Scaling with grid size of the error for the test case of Fig.5 at the location of temperature maximum, for $\chi_{\|} / \chi_{\perp}=10^{3}, 10^{6}, 10^{9}$, for fixed ratio of radial to azimuthal grid points $\left(N_{r} / N_{\theta}=1 / 3\right)$.

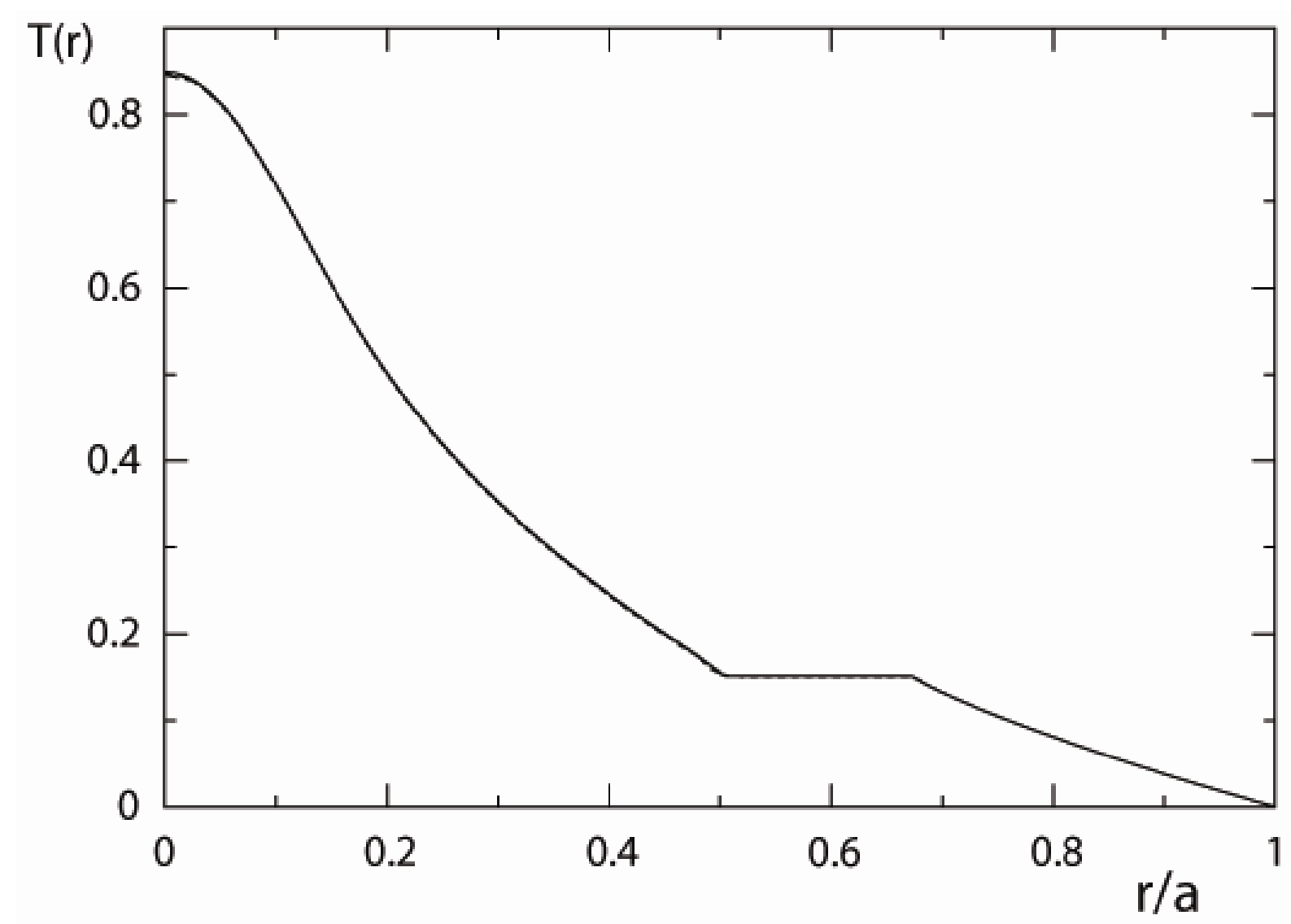

Figure 7: Radial cut of the temperature distribution for a magnetic field configuration with an island, for the $\theta$-value of the O-point $(\theta=0)$, for $\chi_{\|} / \chi_{\perp}=10^{6}$. The results are overlayed for two grid resolutions without meshing, with $N_{r}=N_{\theta}=128$ and 1024, respectively and a case using radial meshing, with $N_{r}=128$ and $N_{\theta}=1024$. 


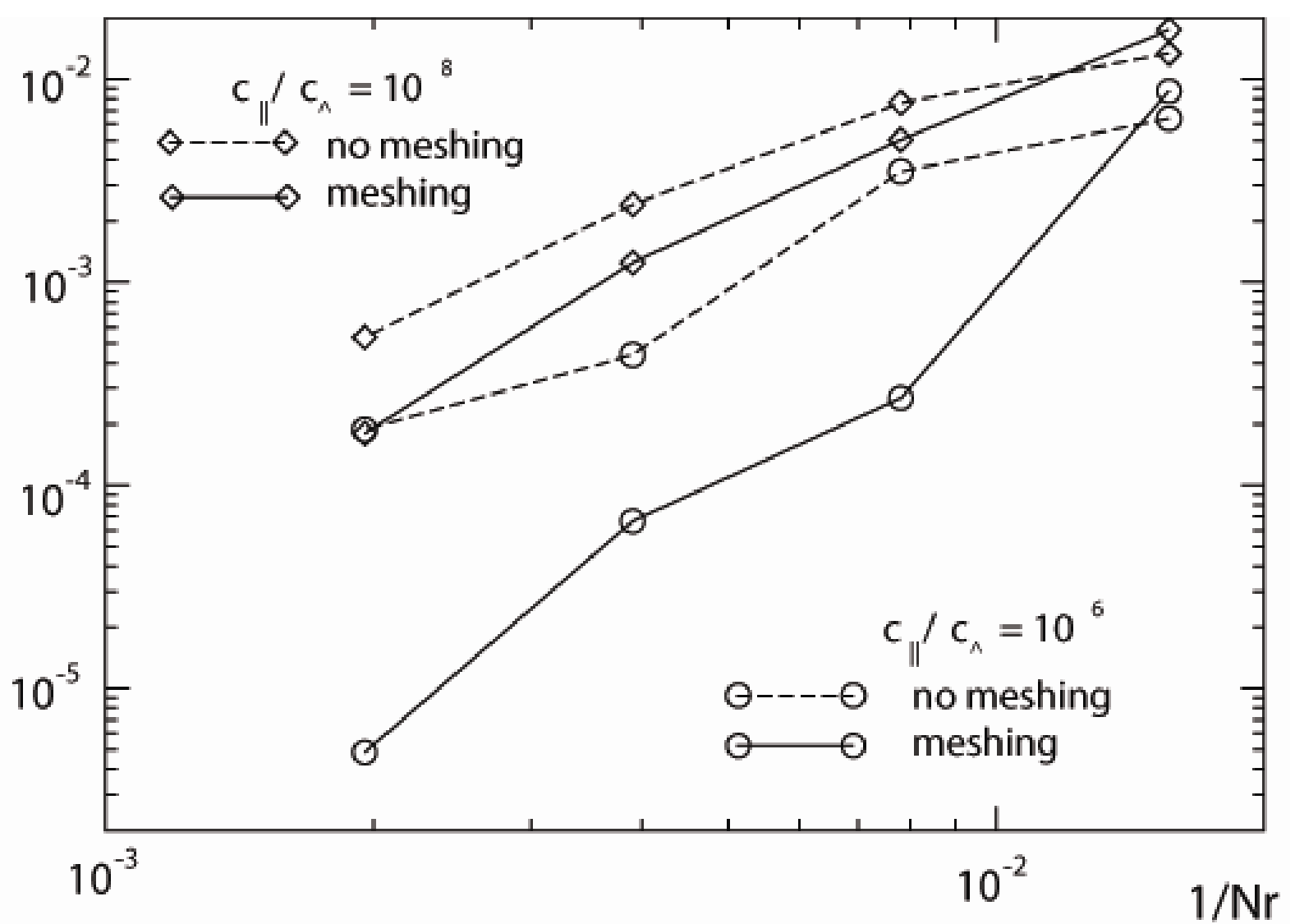

Figure 8: Scaling of error (measured at $r=0.49, \theta=0$, where it is maximum) with radial grid size, for $\chi_{\|} / \chi_{\perp}=10^{6}$ and $10^{8}$, with and without radial meshing, for a constant value of $N_{\theta}=1024$. 


\section{References}

[1] S. Günter et al., J. Comp. Phys. 209 (2005) 354

[2] R. Sovinec et al., J. Comp. Phys. 195 (2004) 355

[3] W. Park et al., Nucl. Fusion 43 (2003) 483

[4] D. Bohm, Minimum kinetic energy for a stable sheath, in The characteristics of electrical discharges in magnetic fields, Eds: A. Guthrie and R.K. Wakerling, Mc Graw Hill, New York 1949

[5] R. Chodura, Phys. Fluids 25 (1982) 1628

[6] F. Wagner and K. Lackner, Divertor tokamak experiments, in Physics of plasma-wall interactions in controlled fusion, Eds: D.E. Post and R. Berisch, Plenum Publishing Corporation 1986

[7] B. J. Braams et al., Contrib. Plasma Phys. 36 (1996) 276

[8] R. Simonini et al., Contrib. Plasma Phys. 34 (1994) 368

[9] T.D. Rognlien et al., Contrib. Plasma Phys. 34 (1994) 362

[10] M. Hölzl et al., Phys. Plasmas 14 (2007) 052501 